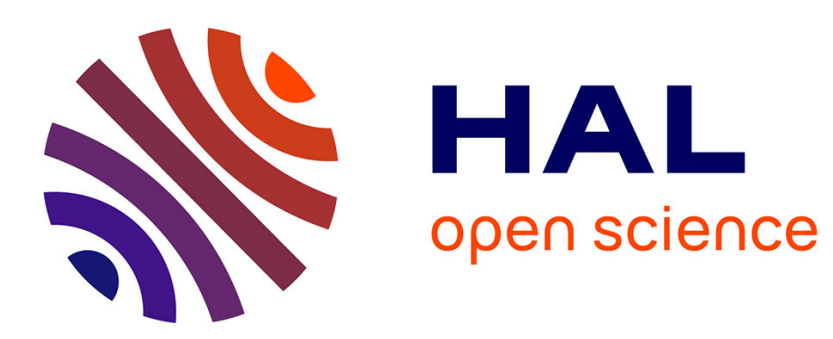

\title{
Piézorésistivité du manganin et de l'ytterbium déposés par pulvérisation cathodique
}

G. Bosca, J. David, L. Allay, J.M. Darmon, Y. Victor

\section{To cite this version:}

G. Bosca, J. David, L. Allay, J.M. Darmon, Y. Victor. Piézorésistivité du manganin et de l'ytterbium déposés par pulvérisation cathodique. Revue de Physique Appliquée, 1981, 16 (7), pp.387-395. 10.1051/rphysap:01981001607038700 . jpa-00244931

\section{HAL Id: jpa-00244931 https://hal.science/jpa-00244931}

Submitted on 1 Jan 1981

HAL is a multi-disciplinary open access archive for the deposit and dissemination of scientific research documents, whether they are published or not. The documents may come from teaching and research institutions in France or abroad, or from public or private research centers.
L'archive ouverte pluridisciplinaire HAL, est destinée au dépôt et à la diffusion de documents scientifiques de niveau recherche, publiés ou non, émanant des établissements d'enseignement et de recherche français ou étrangers, des laboratoires publics ou privés. 


\title{
Piézorésistivité du manganin et de l'ytterbium déposés par pulvérisation cathodique ·
}

\author{
G. Bosca, J. David, L. Allay (*), J. M. Darmon (*) et Y. Victor (*) \\ Commissariat à l'Energie Atomique, Centre d'Etudes de Limeil, B.P. nº 27, 94190 Villeneuve Saint Georges, France \\ (*) Société SEDEME, 9 bis et 11, rue Simonet, 75013 Paris, France
}

(Reçu le 15 septembre 1980, révisé le 26 mars 1981, accepté le 27 mars 1981)

\begin{abstract}
Résumé. - Il est possible de réaliser des jauges piézorésistives de manganin et d'ytterbium par pulvérisation cathodique. Un certain nombre de jauges ont été fabriquées sur différents supports, notamment silice et kapton. Nous présentons les essais effectués pour évaluer leurs coefficients respectifs en pression dynamique.
\end{abstract}

\begin{abstract}
Piezoresistive thin films gauges of manganin and ytterbium can be realized by sputtering deposition. A number of gauges have been made on different substrates, particularly silica and kapton. Experiments for calibration of the dynamic pressure coefficient of both types of gauges are described.
\end{abstract}

1. Introduction. - La mesure de l'amplitude de la pression induite dans un matériau parcouru par une onde de choc peut être faite en utilisant la piézorésistivité de certains métaux.

Le phénomène de piézorésistivité est connu depuis les premières études de Lord Kelvin (1856) et celles de Lisell (1903). Bridgman [1] indique que Lisell étudia un alliage de manganin et qu'en 1909 Lafay, à la suite des travaux de Lisell, étudia la variation de résistivité sous pression de plusieurs échantillons de fils de manganin.

Le manganin $\left({ }^{1}\right)$ est un alliage ternaire de composition variable dont les composants sont :

10 à $15 \%$ de manganèse

2 à $6 \%$ de nickel

le complément de cuivre.

Cet alliage présente l'avantage d'avoir une résistivité variant très peu avec la température et un coefficient piézorésistif $a=\frac{1}{p} \frac{\Delta R}{R}$ positif dont la valeur se situe entre 2,08 et $2,34 \times 10^{-3} / \mathrm{kbar}$ variable suivant l'origine et la fabrication [1] (voir Fig. 1).

Les premières mesures de pression dynamique semblent avoir été réalisées dans les années 1960 par Fuller et Price [2] et [3] avec un matériau dont la composition était $86 \%$ de cuivre, $12 \%$ de manganèse et $2 \%$ de nickel. Le coefficient piézorésistif de ce manganin est $2,1 \times 10^{-3} / \mathrm{kbar}$.

$\left({ }^{1}\right)$ Voir annexe I.
A une époque voisine Bernstein et Keough [4] utilisent du manganin dont la composition est différente : $84 \%$ de cuivre, $12 \%$ de manganèse et $4 \%$ de nickel.

Selon ces deux physiciens il possède un coefficient piézorésistif sous choc voisin de $3,1 \times 10^{-3} / \mathrm{kbar}$. Leur étude [4] est complétée par des mesures du coefficient piézorésistif d'alliages de manganin de diverses origines, notamment celui de Bridgman dont ils donnent la composition : $86 \%$ de cuivre, $10 \%$ de manganèse et $4 \%$ de nickel; et le manganin de Fuller et Price (Fig. 1).

Nous remarquerons que le coefficient piézorésistif qu'ils trouvent pour ce dernier est $2,92 \times 10^{-3} / \mathrm{kbar}$, valeur qui dépasse de $38 \%$ celle que donnent Fuller et Price eux-mêmes.

Lichtenberger [10] montre l'influence du support et des contraintes initiales du manganin. Les résultats diffèrent suivant que la jauge se présente sous forme de fil ou de trame pelliculaire obtenue par photogravure. Il constate que la résistivité d'une feuille de manganin de $6 \mu \mathrm{m}$ passe de $47 \mu \Omega$.cm à $42 \mu \Omega$.cm après un recuit sous vide à $470^{\circ} \mathrm{C}$; cette feuille étant confinée durant le recuit entre deux plaques de manganin bien planes pour éviter l'évaporation du manganèse. La résistivité du manganin en fil est de $43 \mu \Omega . \mathrm{cm}$ après recuit. Le fil de manganin étudié par Lichtenberger a la composition suivante : $86 \%$ de cuivre, $12 \%$ de manganèse et $2 \%$ de nickel, son coefficient piézorésistif est $2,53 \times 10^{-3} / \mathrm{kbar}$. Nous remarquerons 


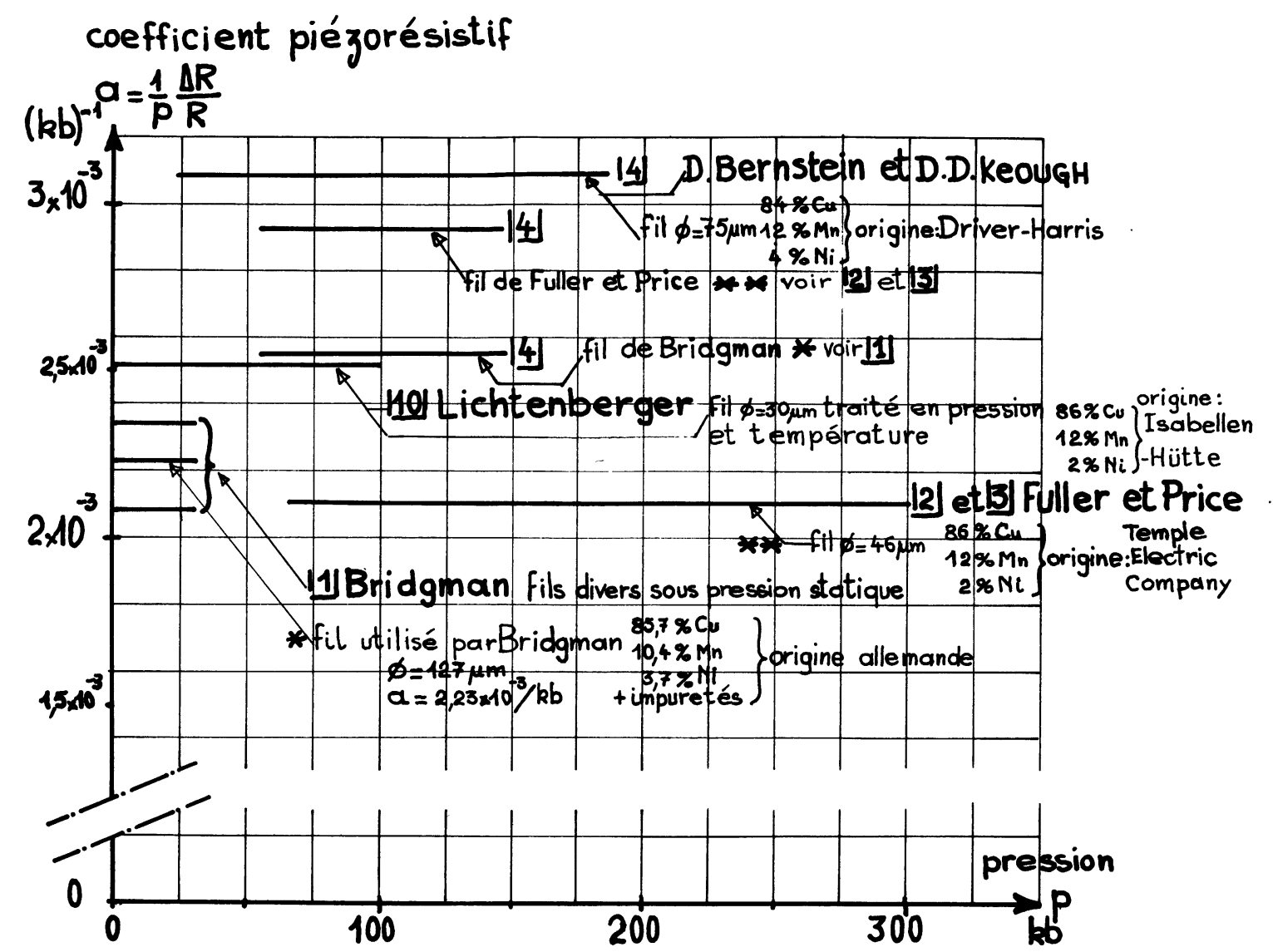

Fig. 1. - Valeur du coefficient piézorésistif de quelques alliages de manganin en fonction de la pression. On notera la diversité des manganins utilisés et la dispersion des coefficients piézorésistifs de compositions identiques notées : ${ }^{*}$ et ${ }^{* *}$.

[Piezoresistive coefficient of some manganin alloys vs. pressure.]

qu'il a la même composition que celui de Fuller et Price.

L'ensemble de ces résultats montrent la diversité des manganins existants et la dispersion des coefficients piézorésistifs trouvés.

L'intérêt de l'ytterbium est évident aux basses pressions dynamiques lorsque l'on voit la variation de la résistance en fonction de la pression que donnent Stromberg et Stephens [5] ou Hall et Merill [13] (voir Fig. 2).

Il a été utilisé principalement par Keough [6] et Ginsberg [7] et C. W. Smith [8] pour mesurer les pressions induites par des ondes de choc dans un milieu géologique soumis à une explosion nucléaire souterraine. Le but de leurs travaux était de réaliser un capteur de pression dynamique fiable et plus sensible qu'un capteur à jauge de manganin dans la gamme de pressions allant de 0 à quelques dizaines de kilobars. Leurs études peuvent être regroupées en deux chapitres : l'un concerne l'élaboration et l'étalonnage de l'élément sensible en ytterbium, l'autre traite de l'influence du support de l'élément sensible sur la mesure à effectuer ainsi que du couplage du capteur au milieu géologique.

L'élément sensible qu'ils emploient est soit une feuille d'ytterbium laminé, soit de l'ytterbium déposé par évaporation.
Comme pour le manganin, les résultats dépendent des conditions d'essais et de l'origine du métal utilisé.

2. Miniaturisation des éléments sensibles piézorésistifs. - Le but principal de toute mesure est de connaître le paramètre mesuré tout en perturbant le moins possible le milieu dans lequel la mesure est faite. La miniaturisation d'un élément piézorésistif, si elle est toutefois réalisable, permettra de perturber au minimum le milieu et de restituer plus fidèlement un des paramètres les plus importants, le front de montée de la mesure s'il s'agit d'une pression produite par une onde de choc.

Un premier pas a été réalisé dans la miniaturisation par Williams [9] avec une jauge de manganin constituée d'une trame pelliculaire dont la résistance a une valeur de $50 \Omega$ pour qu'elle soit adaptée à l'impédance d'un câble coaxial courant.

Lichtenberger [10] a également réalisé des jauges à trame pelliculaire dont la feuille de manganin mesure $6 \mu \mathrm{m}\left({ }^{2}\right)$ et des jauges plates bobinées en fil de manganin de 20 et $30 \mu \mathrm{m}$ de diamètre.

$\left({ }^{2}\right)$ Cette feuille de manganin provenait de la société Kawecki-Billiton Metaal Industrie des Pays-Bas. La composition et l'origine du métal ne sont pas données. 
$R / R_{0} \cdot R$ ésistance électrique relative

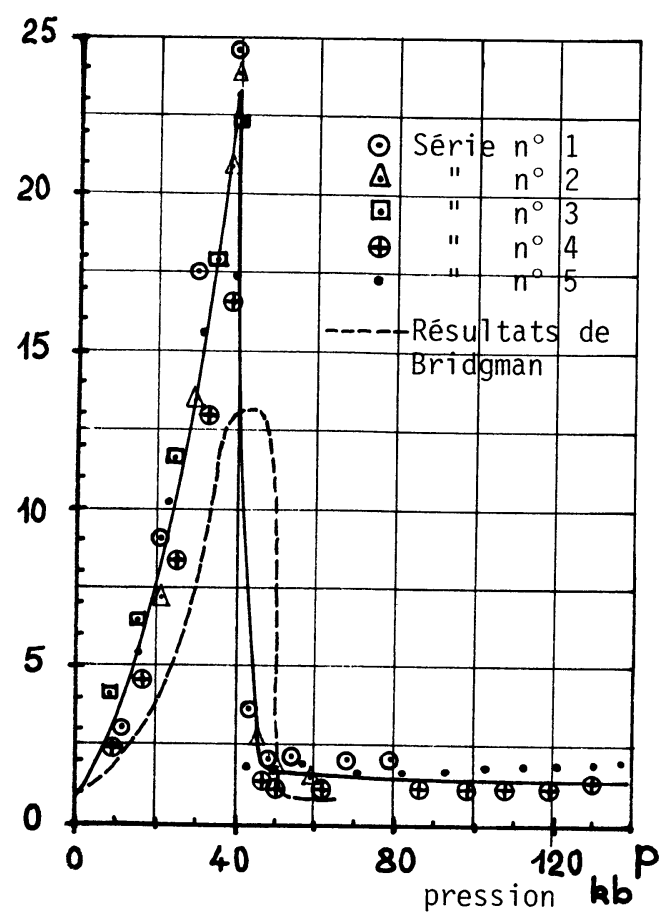

a) Points et courbes de la référence [5]

$R / R_{0}$ Résistance électrique relative

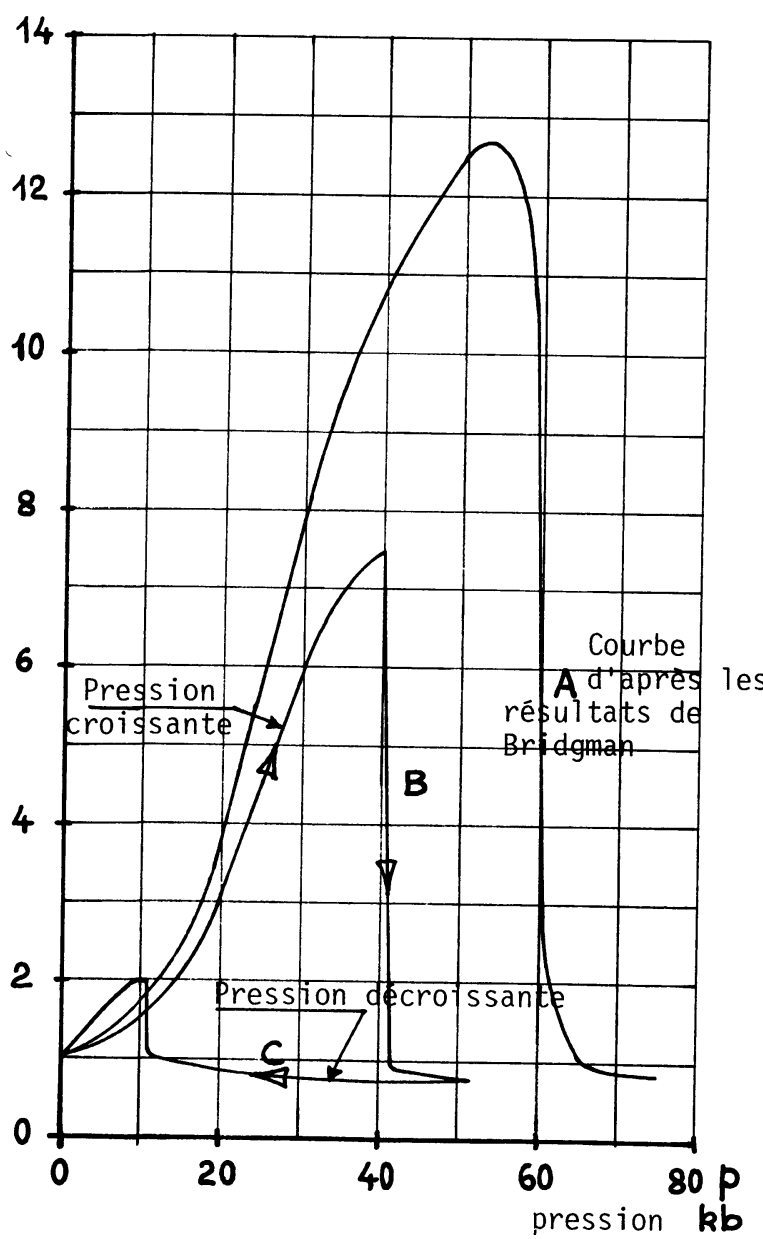

b) Courbes de la référence [13]
Il vient évidemment à l'esprit de celui qui souhaite une miniaturisation plus perfectionnée, de réaliser des jauges par évaporation ou par pulvérisation cathodique. Nous ne sommes pas les premiers à avoir eu cette idée. Des jauges à ytterbium ont été réalisées par évaporation [6].

Malgré les difficultés rencontrées pour réaliser des couches minces d'un alliage ternaire, nous avons étudié la possibilité de fabriquer des jauges de manganin par dépôt. Notre choix s'est arrêté sur la pulvérisation cathodique parce que cette méthode permettait d'envisager de retrouver sur le substrat un dépôt dont la composition serait voisine de la composition initiale de l'alliage ternaire. Nous signalons que dans une publication récente [11] Kannel indique qu'il a réalisé des transducteurs de manganin d'une épaisseur de $0,1 \mu \mathrm{m}$ et $5 \mu \mathrm{m}$ de large sur des galets cylindriques pour étudier les pressions qu'ils subissent au cours de leur fonctionnement. Mais il ne donne pas la valeur du coefficient piézorésistif du dépôt effectué.

3. Jauge manganin déposée par pulvérisation cathodique. - Notre premier travail a été de réaliser des échantillons de manganin pulvérisé sur des substrats de silice et de les analyser pour nous assurer que la composition de l'alliage ne variait pas de façon notable.

Des analyses par spectrométrie d'absorption atomique et par fluorescence $\mathrm{X}$ ont donné pour le manganin massif de la cible :

$80,7 \%$ de cuivre $-5,1 \%$ de nickel

et $14,3 \%$ de manganèse .

L'analyse par fluorescence $X$ de plusieurs échantillons de manganin pulvérisé sur des substrats de silice montre que les pourcentages des différents constituants varient assez peu en fonction des conditions de dépôt.

$$
\begin{array}{ccc}
\text { Cuivre } & \text { Nickel } & \text { Manganèse } \\
77,5 \% \text { à } 82,5 \% & 4,1 \% \text { à } 5 \% & 18,4 \% \text { à } 12,5 \%
\end{array}
$$

Sur la figure 3 nous montrons l'un des échantillons. Son impédance est voisine de $50 \Omega$. Le diamètre du support est de $15 \mathrm{~mm}$. Le dépôt de manganin a la forme du I majuscule situé au centre du disque. Il est relié au circuit électrique de mesure par les deux électrodes d'or déposé en forme de croissant. Une couche de silice de $5000 \AA$ environ est déposée pour protéger l'élément sensible.

L'élément sensible est le barreau mince et étroit dont les dimensions sont $L=6,3 \mathrm{~mm} ; l=100 \mu \mathrm{m}$; $e \sim 6000 \AA$. La résistivité du dépôt est ainsi voisine

Fig. 2. - Variation de résistance de l'ytterbium en fonction de la pression statique.

[Resistance of ytterbium vs. static pressure.] 


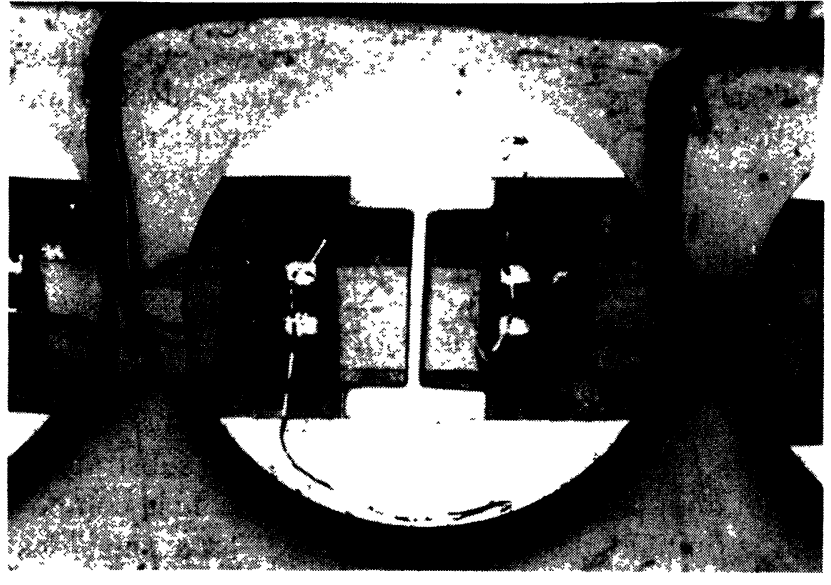

Fig. 3. - Jauge manganin.

[Manganin gauge.]

de $48 \mu \Omega$.cm, valeur très voisine de celle de l'alliage de manganin massif. Son coefficient thermique $\alpha \leqslant 3,2 \times 10^{-5} /{ }^{\circ} \mathrm{C}$ entre $0^{\circ}$ et $130^{\circ} \mathrm{C}$.
Pour l'instant nous n'avons essayé que quelques exemplaires de ces jauges en pression dynamique induite par choc d'un projectile sur une cible. Le schéma du montage est représenté sur la figure 4.

Nous rappelons que la pression induite par un projectile dans une cible de matériau identique à celui du projectile est donnée par l'expression

$$
p=\frac{1}{2} \rho_{0} U v
$$

$\rho_{0}$ étant la masse volumique du matériau avant choc $U$ la vitesse de l'onde de choc qui parcourt le matériau $v$ la vitesse du projectile à l'impact.

Il est ainsi possible de calculer la pression induite en mesurant la vitesse d'onde de choc $U$ et la vitesse $v$ du projectile. La pression induite dans les matériaux accolés à la cible d'aluminium peut se calculer à partir de la méthode dite des polaires de choc décrite par exemple dans les références $[6,17,18]$ traitant de la physique des chocs.

Nous avons réuni les résultats dans le tableau suivant :

\begin{tabular}{|c|c|c|c|c|c|c|c|}
\hline & \multicolumn{4}{|c|}{ Mesures } & \multicolumn{3}{|c|}{ Valeurs calculées } \\
\hline & & & & & & $\ldots$ & $\frac{\Delta R}{D}$ \\
\hline & $v$ & $p$ & $p$ & $\frac{\Delta R}{R}$ & $p$ & $p$ & $a=\frac{R}{n}(\mathrm{~kb})^{-1}$ \\
\hline Essai & Projectile & Quartz & Carbone & manganin & AG3 ou & Araldite & manganin \\
\hline & & & & pulverise & $\begin{array}{c}\text { sllice } \\
\mathrm{kb}\left({ }^{*}\right)\end{array}$ & $\mathrm{kb}\left({ }^{* *}\right)$ & pulvérisé \\
\hline - & - & - & - & - & - & - & - \\
\hline 1 & 165 & 12,4 & 4,8 & $5,1 \times 10^{-3}$ & 12,5 & 4,8 & $1,1 \times 10^{-3}$ \\
\hline 2 & 163 & 12,4 & 4,6 & $6,7 \times 10^{-3}$ & 12,3 & 4,7 & $1,4 \times 10^{-3}$ \\
\hline 3 & 145 & & 5,5 & $24,1 \times 10^{-3}$ & 11,1 & 4,3 & $2,2 \times 10^{-3}$ \\
\hline
\end{tabular}

$\left({ }^{*}\right)$ Calculé à partir de la vitesse du projectile et de la polaire de choc établie par Lundergan [19].

$\left.{ }^{* *}\right)$ On admet que la polaire de choc de l'araldite est identique à celle du plexiglas établie par Halpin [20].

$\left({ }^{* * *}\right)$ Un barreau de silice parallélépipédique recouvrait la jauge qui avait été isolée avec un film d'araldite. La pression prise en compte pour calculer le coefficient piézorésistif est $11,1 \mathrm{~kb}$. L'oscillogramme de cet essai est représenté sur la photo ${ }^{\circ} 1$.

Le résultat du troisième essai est le seul qui soit voisin du coefficient piézorésistif moyen du manganin massif. Un plus grand nombre d'essais nous aurait évidemment permis de connaître l'origine de la distorsion des essais 1 et 2 . Nous ne pouvons formuler que des hypothèses dont la plus simple serait un défaut d'isolement de la jauge. Nous avons remarqué que c'était un paramètre important lors des essais réalisés avec des jauges pelliculaires.

Notre but principal était de montrer qu'il était possible de réaliser des jauges en manganin, ce matériau étant déposé par pulvérisation cathodique, malgré la présence de quelques difficultés techniques.

Il est donc possible, grâce à cette technique de fabrication, d'envisager de déposer des jauges de manganin de petites dimensions sur des substrats très divers.
4. Jauge ytterbium déposée par pulvérisation cathodique. - Apparemment il devrait être plus facile de réaliser des dépôts d'ytterbium parce que c'est un élément simple. Mais l'ensemble des résultats publiés jusqu'à ce jour montre qu'il y a une grande dispersion dans les résultats comme l'indique la figure 5 sur laquelle nous avons porté nos points expérimentaux.

Les jauges que nous avons essayées sont de deux types. Le premier type est réalisé par évaporation du métal chauffé par effet Joule; l'élément sensible du deuxième type est de l'ytterbium déposé par pulvérisation cathodique. La pureté du métal évaporé ou pulvérisé est de $99,9 \%$, sa résistivité est de l'ordre de $30 \mu \Omega . \mathrm{cm}$ à $20^{\circ} \mathrm{C}$. Un vide secondaire inférieur à $10^{-6}$ torr maintenu pendant au moins une heure permet de dégazer le métal. Les substrats sont nettoyés en plusieurs opérations et séchés à l'azote sec peu de 


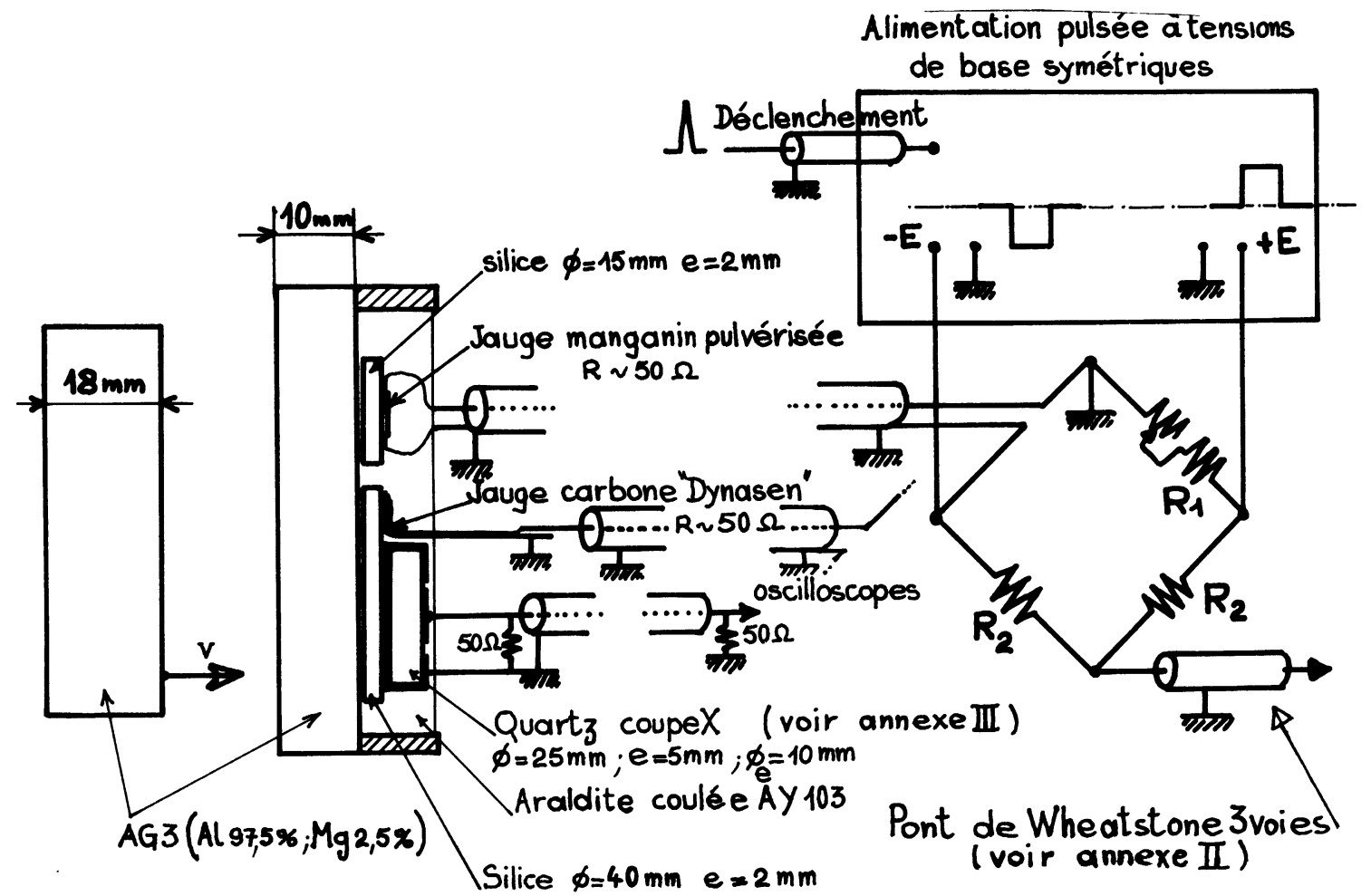

Fig. 4. - Montage expérimental et connexions électriques.

[Experimental assembly and electrical connexions.]

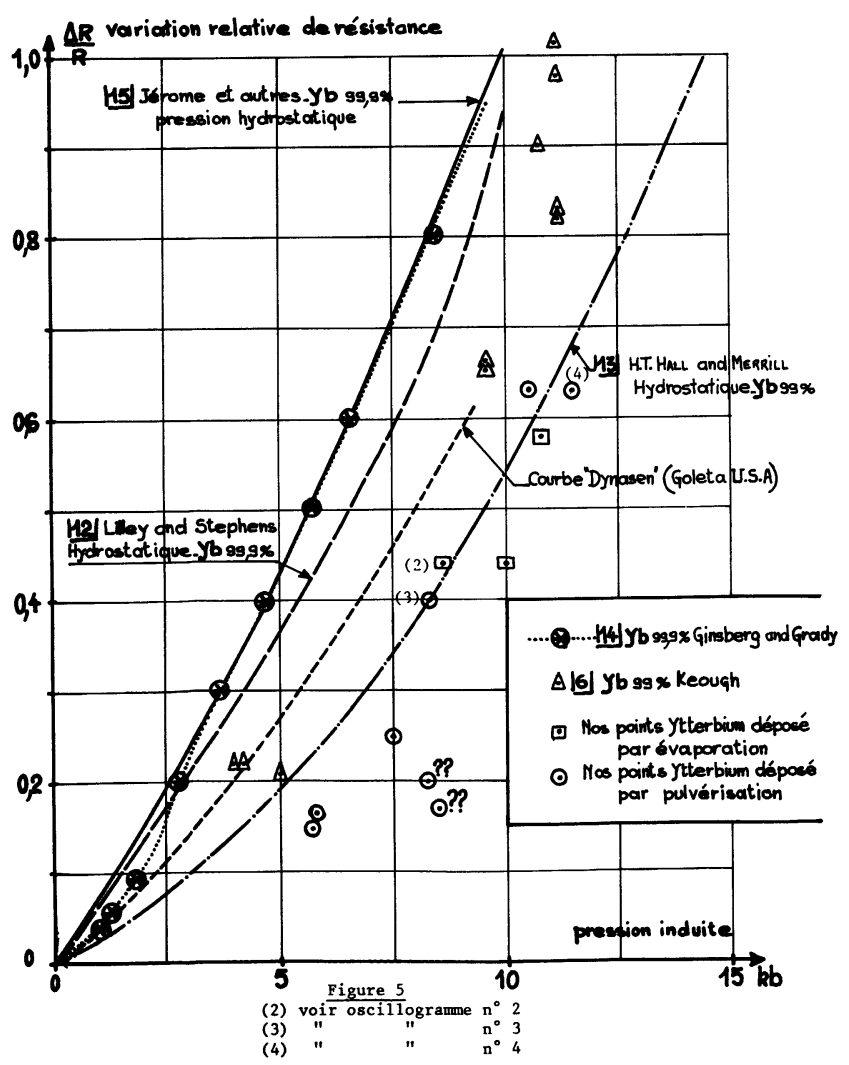

Fig. 5. - Variation relative de résistance de l'ytterbium en fonction de la pression. (2) Voir oscillogramme $n^{\circ} 2$. (3) Voir oscillogramme $n^{\circ}$ 3. (4) Voir oscillogramme $n^{0} 4$.

[Relative variation of resistance of ytterbium vs. dynamic pressure.] temps avant leur mise en place. Comme pour les jauges au manganin les électrodes intermédiaires reliant l'ytterbium aux fils de connexion sont des couches d'or. L'épaisseur des couches déposées est

\section{Signaux des différentes jauges étudiées}

No 1

$0,2 \mathrm{~V} / \mathrm{c}$

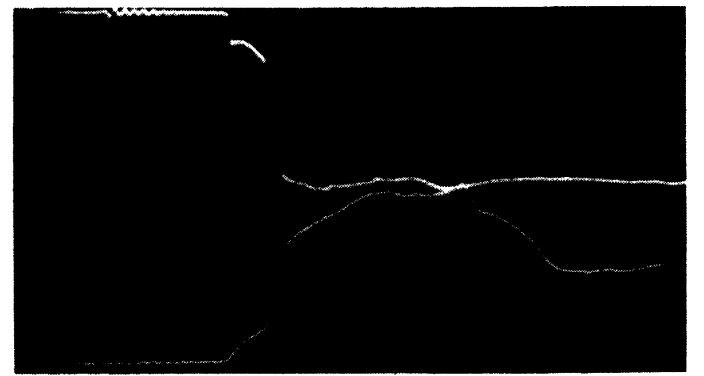

$20 \mathrm{mV} / \mathrm{c}$

$0,5 \mu \mathrm{s} / \mathrm{c}$

Jauge carbone DYNASEN

type FC-300-50-EK

$p=5,5 \mathrm{~kb}$ (araldite)

$v_{\text {projectile }}=145 \mathrm{~m} / \mathrm{s}$

$p_{\text {calculé }}=11,1 \mathrm{~kb}$ dans AG3.

Jauge manganin pulvérisé

$R=52,7 \Omega \frac{\Delta R}{R}=0,024$

essai no 3 (voir tableau).

[Manganin deposited by RF sputtering upper : DYNASEN carbon gauge lower : manganin gauge.] 
N" 2

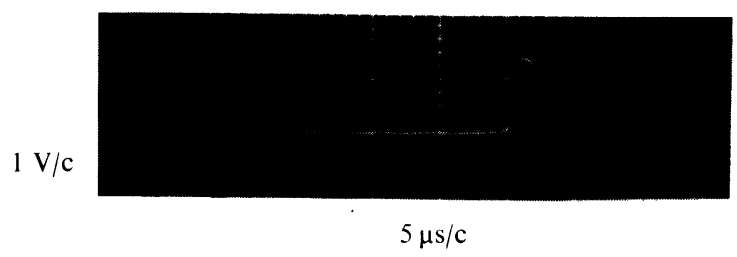

Jauge ytterbium déposé

$R=69 \Omega \frac{\Delta R}{R}=0,43$

$v_{\text {projectile }}=106 \mathrm{~m} / \mathrm{s}$

$p_{\text {calculee }}=8,6 \mathrm{~kb}$

voir point (2) figure 5 .

Le pont de Wheatstone utilisé est différent de celui des autres essais.

[Ytterbium deposited by evaporation.]

No 3

$0,2 \mathrm{~V} / \mathrm{c}$
$0,2 \mathrm{~V} / \mathrm{c}$

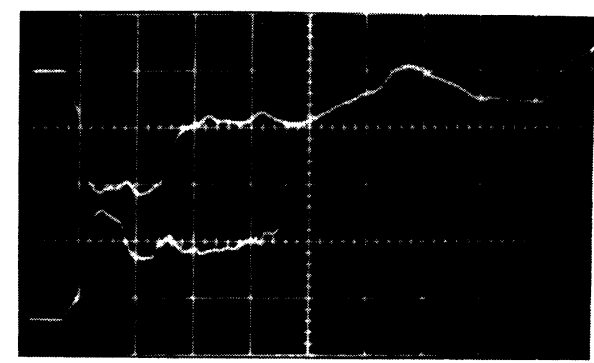

$1 \mu \mathrm{s}$

Jauge carbone $p=8,2 \mathrm{~kb}$

$v_{\text {projectile }}=127 \mathrm{~m} / \mathrm{s}$

$p_{\text {calculee }}=9,2 \mathrm{~kb}$.

Jauge ytterbium pulvérisé $R=50,7 \Omega$

$\frac{\Delta R}{R}=0,40$

voir point (3) figure 5 .

[Ytterbium deposited by RF sputtering upper : carbon gauge

lower : ytterbium gauge.]

No 4

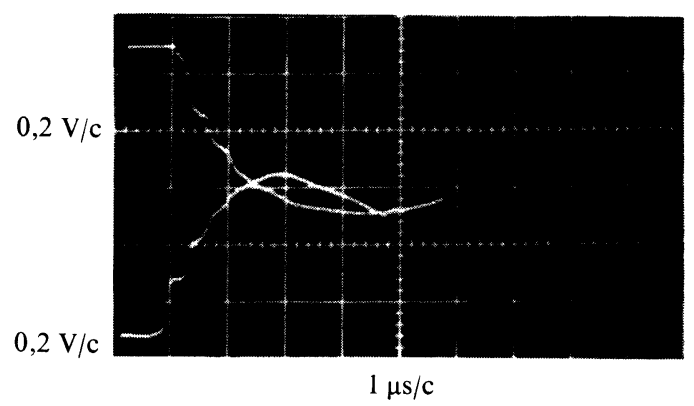

Jauge carbone $p=11,5 \mathrm{~kb}$

$v_{\text {projectile }}=165 \mathrm{~m} / \mathrm{s}$

$p_{\text {calculé }}=12,5 \mathrm{~kb}$.

Jauge ytterbium pulvérisé $R=49,9 \Omega$

$\frac{\Delta R}{R}=0,63$

voir point (4) figure 5 .

[Ytterbium deposited by RF sputtering

upper : carbon gauge

lower : ytterbium gauge.] de quelques milliers d'angströms; elle varie suivant la méthode utilisée pour réaliser le dépôt et la surface totale de la jauge élaborée. La jauge pulvérisée a les dimensions suivantes : $L=6 \mathrm{~mm} ; l=200 \mu \mathrm{m}$; $e \sim 2000 \AA$ pour que la résistance totale soit de l'ordre de $50 \Omega$.

Dans le cas d'ytterbium déposé par évaporation, la résistance de certaines jauges étant plus élevée que celle qui était prévue amène à évaluer la résistivité à une valeur comprise entre 35 et $45 \mu \Omega$.cm.

Les variations de résistivité nous ont amené à protéger les couches d'ytterbium pulvérisé par un dépôt de silice de l'ordre de $5000 \AA$. Une analyse par fluorescence $X$ nous a montré également que le métal déposé par pulvérisation est bien de l'ytterbium avec quelques traces de cuivre. Son coefficient thermique $\alpha \leqslant 3,2 \times 10^{-4} /{ }^{\circ} \mathrm{C}$ entre $25^{\circ} \mathrm{C}$ et $120^{\circ} \mathrm{C}$.

L'ensemble de nos résultats est cohérent à l'exception de deux points isolés dont nous ne savons pas encore expliquer la position. Mais on peut voir que les variations de résistance que nous trouvons sont en moyenne la moitié de celles que donnent Ginsberg, Grady et autres [14].

Il est à noter que l'élément sensible des jauges de Ginsberg est réalisé dans de la feuille d'ytterbium laminé de $50 \mu \mathrm{m}$ alors que nos jauges ont des épaisseurs de quelques milliers d'angströms.

Ginsberg et autres [14] signalent par ailleurs qu'il suffit de recuire le métal laminé pour obtenir des variations de résistance 2 à 3 fois plus importantes que celle du métal laminé. Il faut également se rappeler que l'ytterbium est un métal de la série des terres rares dont la résistivité est élevée pour un métal. Elle est de 25 à $30 \mu \Omega . \mathrm{cm}$, suivant les échantillons. Ginsberg et autres [14] indiquent que certains lots d'ytterbium laminé avaient une résistivité de $30 \mu \Omega$.cm et que l'ytterbium laminé recuit n'avait qu'une résistivité de $19 \mu \Omega . \mathrm{cm}$.

Il semble également que les impuretés du métal et probablement celles qui sont apportées au cours du montage, soit par la colle, soit par la cible, ont une grande influence sur la variation que provoque la pression.

Le diagramme que donnent Hall et Merrill [13] peut résumer partiellement les différences observées entre les divers échantillons.

L'ytterbium devient semi-conducteur si on élève sa pression comme on peut le voir sur la figure 6 . Les travaux de Jérome et autres [15] et ceux de Ribault [16] complètent les travaux de Hall et Merrill.

Au-dessus de 10 à 11,5 kilobars, suivant les auteurs, apparaît le caractère semi-conducteur du métal, qui ne serait total qu'aux environs de 14 à 15 kilobars. Mais dès 5 à 6 kilobars on voit que la pression a une influence importante sur la mobilité des électrons et des trous (voir Fig. 7)

Nous pensons que les irrégularités de fonctionnement d'une même jauge et les divergences apparentes entre les différents types de jauges proviennent de la 


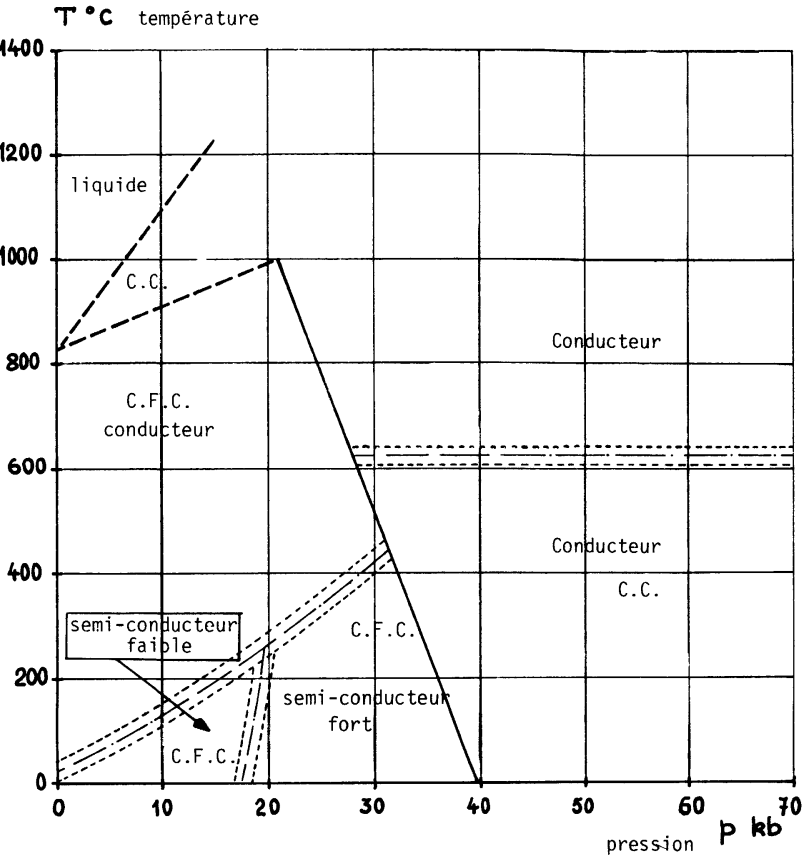

Fig. 6. - Diagramme de phase de l'ytterbium.

[Partial pressure temperature phase diagram for ytterbium.]

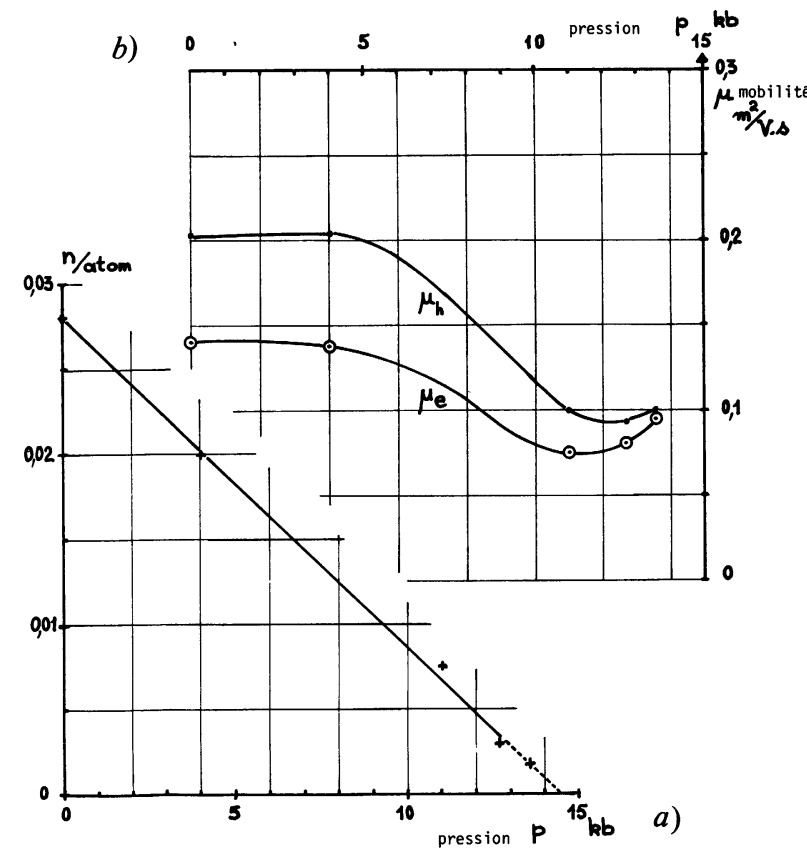

Fig. 7. - a) Concentration des porteurs libres en fonction de la pression. b) Mobilité des électrons et des trous en fonction de la pression.

[a) Pressure dependence of free carriers concentration. $b$ ) Pressure dependence of electrons and holes mobility.]

pureté initiale du métal utilisé, jointe à son caractère bivalent métal-semi-conducteur variable avec la pression et son état électronique réel avant le passage de l'onde de choc.

Récemment Gasgnier et Malaurent [23] et [24] ont montré qu'il est difficile d'obtenir des couches minces d'ytterbium. La réactivité de l'ytterbium avec l'hydrogène, l'oxygène et l'eau conduit à la formation de dihydrure $\left(\mathrm{YbH}_{2}\right)$ et d'oxydes qui modifient d'autant plus les propriétés de la couche, que celle-ci est mince. Ces deux auteurs ont mis en évidence, pour l'ytterbium en couche mince, la coexistence des deux phases hexagonale compacte et cubique à faces centrées entre 77 et $350 \mathrm{~K}$; mais il est difficile de cerner les phénomènes qui régissent leur répartition : température de condensation, impuretés, recuit, trempe... ce qui expliquerait les variations des résultats publiés et celles que nous avons observées.

5. Conclusion. - En conclusion, nous pouvons dire qu'il est essentiel que les utilisateurs de jauges de pression aient conscience de l'importance des caractéristiques propres d'une jauge.

Nos différents essais nous ont montré que l'origine du métal, la technique de fabrication et la technique de montage ont chacune une influence importante sur le fonctionnement de la jauge.

Dans la mesure du possible, il faudrait que chaque laboratoire étalonne les jauges qu'il utilise ou du moins qu'il vérifie que sa technique de montage lui permet de retrouver le coefficient piézorésistif annoncé.

Après quelques études complémentaires et des perfectionnements, nous pensons que les jauges de manganin ou d'ytterbium déposés par pulvérisation cathodique donneraient entière satisfaction dans leurs gammes respectives d'utilisation, à savoir de 0 à 40 kilobars pour l'ytterbium et de 0 à environ 1 mégabar pour le manganin.

Remerciements. - Nous tenons à remercier M. P. Gasiorowski du CEA-Limeil d'avoir réalisé les jauges d'ytterbium déposé ainsi que MM. J. P. Négron et M. Samson du CEA-Limeil pour la réalisation des montages et l'exécution des essais.

Nous tenons également à exprimer nos remerciements à tous les techniciens de la SEDEME qui ont participé à la réalisation des jauges d'ytterbium et de manganin déposés par pulvérisation cathodique.

Il nous est agréable de remercier la Direction de la Revue de Physique Appliquée qui nous a signalé les travaux de MM. Gasgnier et Malaurent qui expliquent certaines irrégularités de fonctionnement de la jauge d'ytterbium.

\section{ANNEXE}

A I. Propos sur l'appellation Manganin. - M. Lichtenberger, auteur de [10], nous a indiqué que la firme Isabellen Hütte de Dillenburg (R.F.A.) vend un alliage breveté sous le nom de manganin contenant $86 \% \mathrm{Cu}$, $12 \% \mathrm{Mn}, 2 \% \mathrm{Ni}$. Nous signalerons que ce mot 
semble être employé pour toutes les compositions voisines comme on peut le voir dans le Nouveau traité de Chimie Minérale de Pascal tome XX, $3^{\mathrm{e}}$ fascicule Alliages Métalliques pages 2138 et 2139 et dans une documentation de la société Gilby-Fodor appelant manganin la composition $84 \% \mathrm{Cu}, 12 \% \mathrm{Mn}$ et $4 \% \mathrm{Ni}$ pour laquelle $\rho=48,1 \mu \Omega \mathrm{cm}$ à $20{ }^{\circ} \mathrm{C}$ et le coefficient thermique est très faible $\alpha=10^{-5} /{ }^{\circ} \mathrm{C}$ entre 0 et $100^{\circ} \mathrm{C}$.

A II. Mesure de pression par jauge piézorésistive. Tout dispositif, capable de suivre la variation de résistance de la jauge, peut être utilisé pour faire la mesure. Dans ces essais, chaque jauge résistive est insérée dans un pont de Wheatstone rapide, capable de détecter une variation relative de résistance de $50 \%$ en 3,5 nanosecondes. La variation relative de la résistance $\Delta R / R$ est reliée à la tension de déséquilibre $\Delta v$ du pont par la formule

$$
\frac{\Delta R}{R}=\frac{24,346 \Delta v}{E-13,761 \Delta v}
$$

qui est calculée en fonction des différentes résistances du circuit de mesure et vérifiée expérimentalement.

La pression $p$ induite dans le matériau est calculée à partir de $\Delta R / R=a \cdot p$ si le coefficient piézorésistif $a$ est connu ou simplement lu sur une courbe fournie par le fabricant de jauge.

A III. Mesure de pression par jauge à quartz. La pression de choc induite dans un quartz (Fig. 1A) [21] et [22] est reliée au courant délivré dans une charge résistive de faible valeur reliant les électrodes par l'expression suivante :

$$
i=k \frac{S U}{e}\left(\sigma_{\mathrm{A}}-\sigma_{\mathrm{B}}\right)=k \frac{S U}{e} \sigma_{\mathrm{q}}
$$

$S$ est la surface de l'électrode centrale,

$e$ est l'épaisseur du quartz,

$U$ est la vitesse de propagation de l'onde de pression dans le quartz,

$\sigma_{\mathrm{A}}$ est la pression exercée sur la face d'entrée A du quartz,

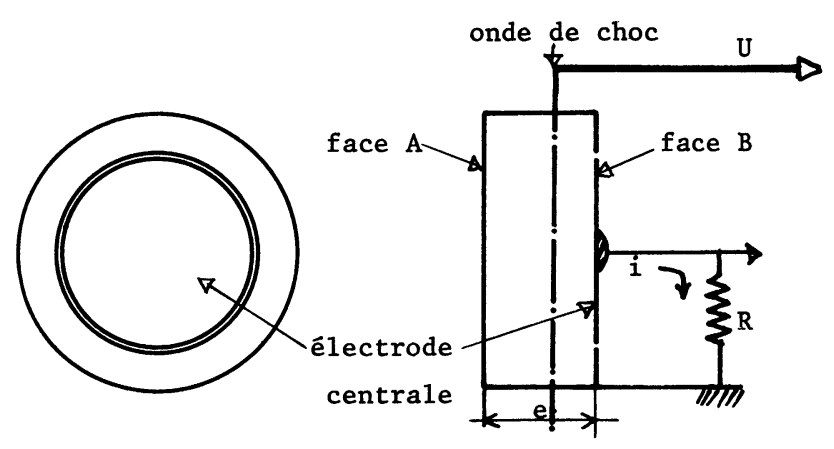

Fig. 1A.
$\sigma_{\mathrm{B}}$ est la pression exercée sur la face de sortie $\overline{\mathrm{B}} \mathrm{du}$ quartz,

$\sigma_{\mathrm{q}}$ est la pression exercée à l'intérieur du quartz à un instant quelconque.

$k$ est le coefficient piézo-électrique dynamique sous choc du quartz. Il dépend de la courbure de l'onde de pression, de la gamme de pressions, du rapport de la surface de l'électrode à la surface totale du disque, ainsi que de l'épaisseur

$$
\begin{array}{rlrl}
k & =2,04 \times 10^{-8} \mathrm{C} / \mathrm{cm}^{2} . \mathrm{kb} & \text { si } & 0<\sigma_{\mathrm{q}}<6 \mathrm{~kb} \\
& =2,15 \times 10^{-8} \mathrm{C} / \mathrm{cm}^{2} \cdot \mathrm{kb} & \text { si } & 6<\sigma_{\mathrm{q}}<25 \mathrm{~kb} \\
\text { d'après [21] }
\end{array}
$$

Signalons que sa valeur est cependant très proche de celle qu'a le coefficient piézo-électrique normal du quartz donné par la relation $Q=d F=d . \sigma . S$ reliant la quantité d'électricité libérée sur les électrodes à la force exercée sur les faces. La valeur de $d$ est $2,3 \times 10^{-12} \mathrm{C} / \mathrm{N}$ ou $2,3 \times 10^{-8} \mathrm{C} / \mathrm{cm}^{2} . \mathrm{kb}$.

En pratique, le signal, délivré par le quartz soumis à une onde de pression dont la durée est supérieure au temps nécessaire à l'onde de choc pour parcourir l'épaisseur $e$, est représenté sur la figure $2 \mathrm{~A}$.
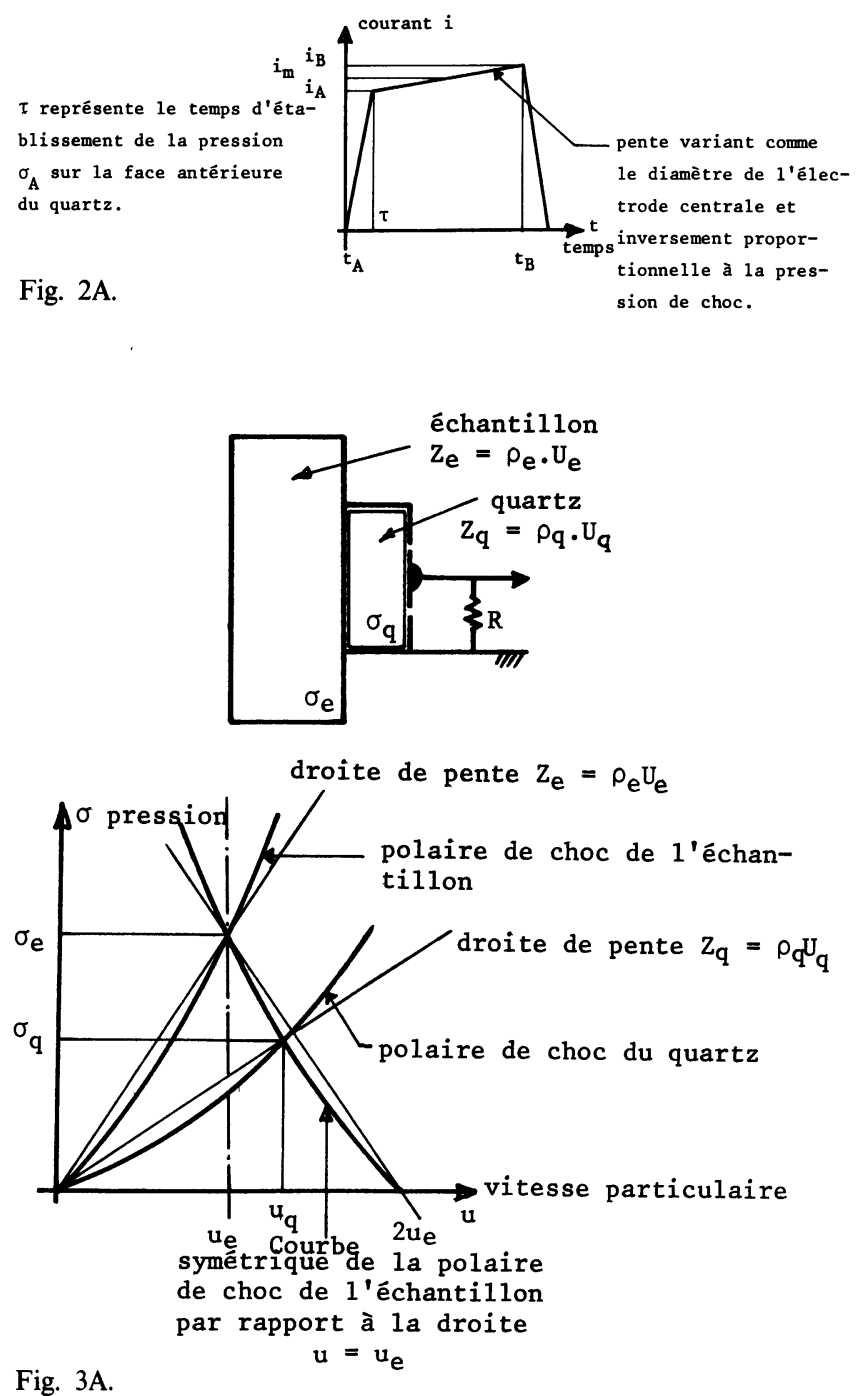

Fig. 3A. 
Des études poursuivies sur des quartz de ce type de différents diamètres et épaisseurs nous ont permis de vérifier la valeur du coefficient $k$ dans la gamme de pressions 0 à $40 \mathrm{~kb}$. Le quartz $« \phi=25 \mathrm{~mm}$; $e=5 \mathrm{~mm} ; \phi_{\mathrm{e}}=10 \mathrm{~mm}$ " relié aux oscilloscopes par un câble coaxial muni à chaque extrémité d'une résistance de $50 \Omega$ (voir Fig. 4) a une sensibilité de $0,45 \mathrm{~V} / \mathrm{kb}$. Pour connaître la pression induite $\sigma_{\mathrm{e}}$ dans un échàntillon à partir de la pression induite dans le quartz qui lui est accolé (Fig. 3A), il est nécessaire de connaître la polaire de choc ou l'impédance de $\operatorname{choc} Z_{\mathrm{e}}$ de l'échantillon pour calculer la pression $\sigma_{\mathrm{e}}$ à partir de la valeur mesurée $\sigma_{\mathrm{q}}$.
Aux basses pressions dynamiques en régime élastique, on a la relation obtenue par similitude de triangles

$$
\sigma_{\mathrm{e}}=\frac{Z_{\mathrm{e}}+Z_{\mathrm{q}}}{2 Z_{\mathrm{q}}} \cdot \sigma_{\mathrm{q}}
$$

Pour avoir plus de précision, dès que la courbure de la polaire de choc est importante, il est préférable d'utiliser les polaires de choc de l'échantillon et du quartz pour déterminer la pression $\sigma_{\mathrm{e}}$ par construction graphique ou calcul.

\section{Bibliographie}

[1] Bridgman, P. W., The Physics of High Pressure. (G. Bell and Sons Ltd, London) 1958.

[2] Fuller, P. J. A., Price, J. H., Electrical Conductivity of Manganin and Iron at High Pressures. Nature Vol. 193 (1962).

[3] Fuller, P. J. A., Price, J. H., Dynamic pressure measurements to 300 kilobars with a resistance transducer, Brit. J. Appl. Phys. Vol. 15 (1964).

[4] Bernstein, D., KeOugh, D. D., Piezoresistivity of Manganin, J. Appl. Phys. Vol. 35 (1964) 1471-1474.

[5] StromberG, H. D., STHEPHENS, D. R., Effects of Pressure on the Electrical Resistance of certain metals. J. Phys. Chem. Solids (Pergamon Press) Vol. 25 (1964) 1015-1022.

[6] Keough, D. D., Development of a high sensitivity piezoresistive shock transducer for the low kilobar range, March 25 (1970) Final Report DASA 2508.

[7] Ginsberg, M. J., Calibration and Characterization of Ytterbium stress transducers, Final Report DNA 2742 F, October (1971).

[8] SмIтH, C. W., Dynamic measurements of stress pulses in tuff at Nevada Test Site, Final Report ; DNA 2811 F, August (1971).

[9] Williams, E. O., An Etched Manganin gauge system for pressure measurement in a high-noise environment, ISA Transactions, Vol. 7 (1968) 223-230.

[10] Lichtenberger, A., Contribution à l'étude des capteurs piézorésistifs minces à base de manganin. Application aux ondes de choc, Thèse de Docteur-Ingénieur présentée à la Faculté des Sciences et des Techniques de Besançon le 6 octobre 1973, Rapport 16/73 du 17 mai 1973, Institut FrancoAllemand de Saint-Louis.

[11] Kannel, J. W., Vapor Deposited Transducers for measuring pressure and temperature in machine elements, Design Engineering Conference and Show of A.S.M.E., Chicago, May 9-12 1977 ASME paper 77 DE 33.

[12] LiLley, E. M., StePhens, D. R., Electrical resistance of Ytterbium as a function of temperature and pressure, 1971, UCRL-51006-TID-4500, UC.4 Chemistry.
[13] Hall, H. T. and Merrill, L., Some high pressure studies on Ytterbium. - Inorg. Chem. Vol. 2 (1963) 618.

[14] Ginsberg, M. J., Grady, D. E., De Carli, P. S. and RosenBERG, J. T., Effects of Stress on the electrical resistance of Ytterbium and calibration of Ytterbium stress transducers, August 1973, Report DNA no 3577 F.

[15] Jerome, D., RieuX, M., ACHARD, J. C., Ytterbium under pressure. Les propriétés des solides sous pression, Grenoble 8-10 septembre 1969, Editions du CNRS 1970.

[16] Ribault, M., L'effet de Haas-Van Alphen dans l'ytterbium C.F.C. sous pression, Thèse de Doctorat ès Sciences Physiques soutenue le 21 février 1975. Ann. Phys. t. 2, pp. 53-90.

[17] Berger, J., Viard, J., Physique des explosifs solides. (Librairie Dunod) 1962.

[18] JaUmotte, A. L., et autres, Chocs et ondes de choc. (Masson et Cie Editeurs) 1971.

[19] Lundergan, C. D. and Herrmann, W., Equation of State of 6061-T6 Aluminium at low pressures, J. Appl. Phys. Vol. 34 (1963) 2046-2052.

[20] Halpin, W. J. and Graham, R. A., Shock wave compression of plexiglas from 3 to 20 kilobars, Sandia Laboratory, Albuquerque, New-Mexico. 4th Symposium on Detonation, 12-15 October 1965, pp. B 38-B 46.

[21] Graham, R. A., Neilson, F. W. and Benedick, W. B., Piezoelectric current from shock-loaded quartz : a submicrosecond stress gauge. J. Appl. Phys. Vol. 36 (1965) 1775 1783.

[22] Graham, R. A., Piezoelectric current from shunted and shorted guard ring quartz gauges. J. Appl. Phys. Vol. 46, $\mathrm{n}^{\circ} 5$ (1975).

[23] Gasgnier, M. et Malaurent, J. C., Etudes de couches minces d'ytterbium par diffraction X in situ, Journal Appl. Crystallogr. $\mathrm{n}^{\circ} 11$ (1978) pp. 141-147.

[24] GASGNIER, M., Rare earth metals, rare earth hydrides and rare earth oxides as thin films. A critical review. Phys. Status Solidi A Vol. 57 no 1. 